\title{
Aus einem Schreiben des Herrn Geheinerath A. Auwers an den Herausgeber.
}

Mit Bezugnahme auf die kurzlich in den A. N. vorgekommenen Aufforderungen zur Einsendung correspondirender Beobachtungen von Mondculminationen oder Sternbedeckungen aus dem Jahre $1882 / 83$ erlaube ich mir mitzutheilen, dass nach meinen über die Monate Sept. I 882 bis Januar 1883 ausgedehnten Rechnungen die mit Newcomb's Correctionen verbesserten Hansen'schen Tafeln die Gesammtheit der mir zugänglich gewordenen Beobachtungen so gut wie vollkommen darstellen. Nahe 300 Culminationen ats dem genannten Zeitraum geben eine kleine negative Restcorrection der AR., etwa halb so viel Bedeckungen eine kleine positive.

Für die längenbestimmungen des internationalen Polar-
Unternehmens wird es daher nicht allein ausreichen, die angestellten Beobachtungen mit den nach Newcomb verbesserten Tafeln zu vergleichen, sondern hiervon sogar ein wesentlich genaueres Resultat zu erwarten sein, als wenn man, wie es zu den Zeiten der Burckhardt'schen 'Tafeln ganz angemessen war, mit isolirten correspondirenden Beobachtungen vergleichen wollte.

Für eine eingehendere Mittheilung iiber die Fehler der Mondtafeln $1882 / 83$ muss ich den Abschluss der Untersuchungen abwarten, welche in Betreff einiger der benutzten Sternbedeckungsreihen noch wïnschenswerth sind. Ich hoffe, die dazu nöthigen Daten im Laufe der nächsten Monate zu erhalten.

A. Auncrs.

\section{Planet (238) Hypatia, beobachtet an 9 zölligen Refiactor der Berliner Sternwarte.}

\begin{tabular}{|c|c|c|c|c|c|}
\hline I 884 & M. Z. Berlin & $a$ app. & $\delta$ арр. & Gr. & Vergleichstern \\
\hline Juli 26 & $12^{\mathrm{i}} 5^{8^{\mathrm{m}}} 27^{\mathrm{s}}$ & $22^{h_{1}} 43^{w_{1}} 24^{5}$ I 5 & $+3^{\circ} 2^{\prime} 5^{8.4}$ & I 1.7 & Weisse 805 \\
\hline 26 & 134259 & - & +3253.8 & I I. 7 & Weisse 805 \\
\hline $3^{\circ}$ & I 3 I I 59 & - & $+2505^{8.6}$ & I 1.5 & Weisse 8 ro \\
\hline 30 & 131929 & $2241 \quad 59.05$ & - & I I .5 & Weisse 810 \\
\hline 30 & I 35 I 59 & $2241 \quad 58.19$ & +25054.7 & I 1.5 & Weisse 810 \\
\hline 30 & 14749 & $2241 \quad 58.00$ & +25052.2 & I I. 5 & Weisse 810 \\
\hline
\end{tabular}

Oerter der Vergleichsterne.

Juli 26 W. $805-$ Sj. $9299 \quad \Delta a=+1^{111} 33^{\text {s. }} 5$ Ad $A d=-7^{\prime} 39^{\prime \prime} 9$ ro Beobachtungen.

$30 \mathrm{~W} .810-\mathrm{W} .805 \quad \Delta c=+016.40 \Delta \delta=-320.4 \quad 5$ Beobachtungen.

Mittl. Ort von Sj. 9299 für $1884.0: \quad a=22^{\mathrm{h}} 37^{\mathrm{m}} 54^{\mathrm{s}} .88 d=+3^{\circ}$ I $2^{\prime} 29^{\prime \prime} 3$.

Die $A \alpha$ und $A \delta$ sind für Refraction corrigirt. Die benutzte Vergrösserung betrug $\mathbf{r}_{4} \mathrm{O}$.

Berlin 1884 Aug. 2.

Dr. A. Marcuse.

\section{Beobachtungen des Planeten (240) in Algier.}

(Telegramm an die Centralstelle.)

\begin{tabular}{|c|c|c|c|}
\hline 1884 & 'I. M. Alger & AR. app. & DP. app. \\
\hline $\begin{array}{l}\text { Août } 29 \\
\text { Sept. I }\end{array}$ & $\begin{array}{l}11^{\mathrm{h}} 52^{\mathrm{m}} \cdot 8 \\
\text { IO } 59.1\end{array}$ & $\begin{array}{lll}33^{\circ} & 47^{\prime} & 19^{\prime \prime} \\
338 & 8 & 58\end{array}$ & 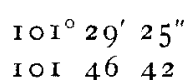 \\
\hline
\end{tabular}

Trépied.

Diese Beobachtungen wurden den Theilnehmern der Centralstelle durch Circular mitgetheilt. Kr.

\section{In halt:}

Zu Nr. 2612. HI. Seeliger. Zur Photometrie des Saturnringes, 305. - H. Pomerantzeff. Ueber Beobachtungen von Sternschmuppen zu Taschkent am 23. und 24. Juni 1884 und ihre Beziehung zu dem Cometen I8501. 315. - Trípiex. Beobachtungen des Cometen I 884 Bamard auf der Sternwarte zu Algier. 317. - Perrotin. Observations de la comète Barnard faites à l'Observatoire de Nice. 317. - A. Autrers. Schreiben an den Herausgeber. 319. - A. Marcuse. Ilanet (238) Hypatia, beobachtet am 9 zölligen Refractor der Berliner Sternwarte. 319. - Tripied. Beobachtungen des Planeten (240) in Algier. 3 I 9. 